\title{
The Three-dimensional Constitution of Polymeric Micelles as Studied by Cryo Electron Microscopy and Tomography
}

\author{
Natalia Chebotareva*, Paul H.H. Bomans, ${ }^{* *}$ Felix de Haas, ${ }^{* * *}$ Dominicus H.W. Hubert, ${ }^{* * *}$ Peter M. \\ Frederik, ${ }^{* *}$ Rint P. Sijbesma, * Nico A.J.M. Sommerdijk ${ }^{*}$ \\ *Eindhoven University of Technology, Laboratory of Macromolecular and Organic Chemistry, PO \\ 513, $5600 \mathrm{MB}$, Eindhoven, the Netherlands \\ **Maastricht University, EM unit, Pathology, PO 616, 6200 MD Maastricht, the Netherlands \\ ***FEI Company, NanoPort, Building AAE, PO 80066, 5600 KA, Eindhoven, the Netherlands
}

Self-assembled micelles in block copolymers have been subjected to a study using well established techniques in nano-biology. Cryo-Electron Microscopy developed in the late 1980's [1] has now become the best technique in Biology to record structural details of proteins, enzymes, viruses and cellular substructures at sub nanometer resolutions without artifacts from specimen preserving/staining agents.

Development in automation at the level of data acquisition on high standard transmission electron microscopes has evolved more and more since the mid 90's[2-4]. Automated acquisition of tomograms now become a standard technique in electron microscopy $[5,6]$. Both techniques combined, Cryo-EM and Tomography, result in tomograms which, when processed toward a threedimensional representation reveal the 3D organization of the material preserved to the 6-3 nm detail.

A dispersion of block copolymers in water will mainly consist of micelles. The more a-polar tails will form a hydrophobic core with on the surface a corona comprising the more hydrophilic part of the block copolymer [7]. Diluted solutions of low molecular weight surfactants have been considered to comprise stretched and fully hydrated monomeric molecules. In more concentrated solutions reaching the critical micelle concentration (CMC), micelles emerge next to the monomeric entities. However, surface tension measurements [8] and gel filtration chromatography [9] have indicated also the formation of oligomers of surfactants below CMC.

The study visualizes the formation of aggregates smaller than micelles for a polymeric surfactant. Image processing of single views of the micelles and pre-micellar aggregates (PMAs) show their dimensions (micelles $43 \mathrm{~nm}$, micellar cores 20nm, PMA's 20nm).

Tomography subjected to three-dimensional reconstruction reveals that PMA's tend to sit at the vacuum-ice interface spanned up by the thicker micelles. As is found by virus cryo-TEM work the material tends to reorganize itself by size exclusion at the air-water interface during thin film formation at room temperature, just before plunging into the cryo-coolant.

\section{References}

[1] Dubochet J, A.M., Chang J. J., Homo J. C., Lepault J., Mcdowall A. W., Schultz P., Quart. Rev. Biophys. 21 (1988) 129.

[2] Dierksen, K.T., D. Hegerl, R. Koster, A. J., Baumeister W., Ultramicroscopy 40 (1992) 71.

[3] Koster, A.J.C., H. Sedat, J. W., Agard, D. A., 46 Ultramicroscopy (1992) 207. 
[4] Braunfeld, M.B., Koster, A.J., Sedat, J.W. and Agard, D.A., J Microsc 174 (1994), 75.

[5] Baumeister, W., FEBS Lett 579 (2005), 933.

[6] Baumeister, W. and Steven, A.C., Trends Biochem Sci 25 (2000) 624.

[7] Won, Y.-Y.; Brannan, A. K.; Davis, H. T.; Bates, F. S., J. Phys. Chem. B 106 (2002), 3354.

[8] Menger, F.M. Littau, C.A., J. Am. Chem. Soc. 115 (1993), 10083.

[9] Funasaki, N. Shim, H.-S. Hada, S.,J. Phys. Chem. 96 (1992), 1998. 